\title{
Impact of ablator thickness and laser drive duration on a platform for supersonic, shockwave-driven hydrodynamic instability experiments
}

\author{
W.C. $\mathrm{Wan}^{1 *}$, G. Malamud ${ }^{1,2 *}$, A. Shimony ${ }^{2,3}$, C.A. Di Stefano ${ }^{1,4}$, M.R. \\ Trantham $^{1}$, S.R. Klein ${ }^{1}$, J.D. Soltis ${ }^{1}$, D. Shvarts ${ }^{1,2,3}$, R.P. Drake ${ }^{1}$, C.C. Kuranz ${ }^{1}$ \\ ${ }^{1}$ Department of Climate and Space Science and Engineering, \\ University of Michigan, USA \\ ${ }^{2}$ Department of Physics, \\ Nuclear Research Center - Negev, Israel \\ ${ }^{3}$ Department of Physics, \\ Ben Gurion University of the Negev, Israel \\ ${ }^{4}$ Los Alamos National Laboratory, USA
}

(Dated: October 25, 2016)

\begin{abstract}
We discuss changes to a target design that improved the quality and consistency of data obtained through a novel experimental platform that enables the study of hydrodynamic instabilities in a compressible regime. The experiment uses a laser to drive steady, supersonic shockwave over well-characterized initial perturbations. Early experiments were adversely affected by inadequate experimental timescales and, potentially, an unintended secondary shockwave. These issues were addressed by extending the $4 \times 10^{13} \mathrm{~W} / \mathrm{cm}^{2}$ laser pulse from $19 \mathrm{~ns}$ to $28 \mathrm{~ns}$, and increasing the ablator thickness from $185 \mu \mathrm{m}$ to $500 \mu \mathrm{m}$. We present data demonstrating the performance of the platform.
\end{abstract}

\section{INTRODUCTION}

Hydrodynamic instabilities play a dominant role in the transport of mass, momentum, and energy in nearly all plasma environments, including the corona of the sun [1], magnetospheric boundaries [2], and astrophysical jets $[3,4]$. In past decades, the contribution of these processes to the intermixing of fluids was underestimated in high-energy-density systems such as core-collapse supernovae $[5,6]$ and fusion experiments $[7,8]$. Since then, significant improvements have been made to experimental techniques, diagnostics, and simulation codes. Here, we discuss an experimental platform that can sustain a steady, supersonic flow over a precision-machined, wellcharacterized material interface for unprecedented durations.

This platform combines the strengths of two major techniques currently used to study hydrodynamic instabilities. Shock tube experiments can sustain steady, supersonic flows for long durations, but rely on statistical assumptions about the initial conditions at the material interface [9-11]. Laser-driven experiments can seed tightly-controlled perturbations into the material interface and obtain astrophysically relevant temperatures and pressures, but are limited by small data sets and, previously, have relied upon blast waves to drive the growth of the hydrodynamic instabilities $[12,13]$. This laserdriven platform creates a shockwave instead of a blast wave using an 19 to $28 \mathrm{~ns}$ laser pulse, driving the evolution of the instabilities with a different mechanism and resulting in a nominally time-invariant velocity that remains supersonic long enough for effects of compressibility to measurably impact the evolution of the instability [14].

This platform has been used to study the RichtmyerMeshkov instability [15], the Kelvin-Helmholtz instability $[14,16]$, and their mutual interaction. For the purpose of this discussion, we will focus on early experiments investigating the Kelvin-Helmholtz instability. The Kelvin-Helmholtz instability is a process can produce vortical structures and turbulence in a system of fluids with strong velocity shear $[17,18]$. In a supersonic flow, the instability growth is inhibited and the resulting structure is altered $[14,19]$. This inhibition of the Kelvin-Helmholtz vortex growth rate, along with the two-vortex merger rate, are the core components needed to develop a comprehensive model for the evolution of the Kelvin-Helmholtz instability in a compressible flow, such as one would find in many astrophysical and engineering systems [1-8]. Both aspects are still poorly understood, and hold the motivation for the current work. The experimental platform discussed here provides, for 
the first time, the capabilities to diagnose the effects of compressibility on the evolution of hydrodynamic instabilities from well-controlled seed perturbations.

This platform produced the first experimental observations of a well-characterized interface evolving under the effects of the Kelvin-Helmholtz instability in a steady, supersonic flow [16]. Obtaining these data required adjustments to optimize the original target design.

\section{TARGET DESIGN AND METHODOLOGY}

This experimental platform utilized the OMEGA-EP facility to produce a steady, supersonic flow across a wellcontrolled and well-characterized seed perturbation. The OMEGA-EP facility is unique in the world for having four independently operated, highly energetic lasers [20]. For the purpose of these experiments, the target is irradiated with two to three lasers at a wavelength of $351 \mathrm{~nm}$. Each of these beams contained 4 kJ of energy in a $10 \mathrm{~ns}$ square pulse, except for a 1 ns transitioning ramp as one laser pulse ends and another, overlapping pulse begins. This $1 \mathrm{~ns}$ transition period mitigates any potential problems from laser timing errors, and results in a nominally 19-28 ns square pulse, over a $1.1 \mathrm{~mm}$ diameter spot size, with an average intensity $\sim 4 \times 10^{13} \mathrm{~W} / \mathrm{cm}^{2}$. Currently, OMEGA-EP is the only facility capable of producing this combination of high-energies and long pulse durations. The extended laser pulse maintains pressure on the drive surface in order to sustain a strong, steady shock through the system at maximum compression. This results in a system that can seed instability growth with tightly controlled initial perturbations in a nominally time-invariant supersonic flow.

Figure 1 shows a pre-experimental x-ray radiograph of a sample target. Starting from the left, we have a polycarbonate $(\mathrm{PC})\left(\mathrm{C}_{16} \mathrm{H}_{14} \mathrm{O}_{3}, \rho=1.1 \mathrm{~g} / \mathrm{cm}^{3}\right)$ ablator. The lasers are incident upon this layer, and generate the initial shockwave. In the original experiment, the ablator was $185 \mu \mathrm{m}$ thick and irradiated with a $19 \mathrm{~ns}$ laser drive. In the subsequent experiment [16], the ablator was increased to $500 \mu \mathrm{m}$ and irradiated with a $28 \mathrm{~ns}$ drive. The PC ablator is followed by a $100 \mu \mathrm{m}$ thick layer of brominated plastic thermal insulator $(\mathrm{CHBr})\left(\mathrm{C}_{50} \mathrm{H}_{48} \mathrm{Br}_{2}, \rho=\right.$
$1.2 \mathrm{~g} / \mathrm{cm}^{3}$ ), which acts as a heat shield to prevent preheating of the materials that follow. The remainder of the target can be described as the "physics package," and can be divided into a top half and a bottom half.

The top layer of the physics package is carbonizedresorcinol-formaldehyde foam $(\mathrm{CRF})\left(\mathrm{C}_{1000} \mathrm{O}_{48} \mathrm{H}_{65}, \rho=\right.$ $\left.0.10 \mathrm{~g} / \mathrm{cm}^{3}\right)$, which acts as our low-density fluid. Beneath it is an iodinated polystyrene tracer strip (CHI) $\left(\mathrm{C}_{50} \mathrm{H}_{47} \mathrm{I}_{3}, \rho=1.4 \mathrm{~g} / \mathrm{cm}^{3}\right)$ embedded into a densitymatched polyamide-imide block (PAI) $\left(\mathrm{C}_{22} \mathrm{H}_{14} \mathrm{O}_{4} \mathrm{~N}_{2}, \rho=\right.$ $1.4 \mathrm{~g} / \mathrm{cm}^{3}$ ), which together act as our high-density fluid. The iodinated plastic provides a narrow image plane that absorbs the majority of the $\mathrm{Cu}$ x-rays, while the surrounding polyamide-imide is highly transmissive to the $\mathrm{Cu}$ x-rays; this mitigates potential smearing of smallscale structure due to misalignment or edge effects. A 100 $\mu \mathrm{m}$ wavelength, $5 \mu \mathrm{m}$ amplitude single-mode sinusoidal perturbation is machined into the surface of this plastic. Finally, a gold block rests between the thermal insulator and the heavier fluid, which slows down the lower half of the incoming shockwave such that it does not reach the plastic behind it on experimental timescales. This shock blocker maximizes our relative velocity and provides a clean start to the experiment, when the supersonic shear flow is first introduced over the machined perturbation.

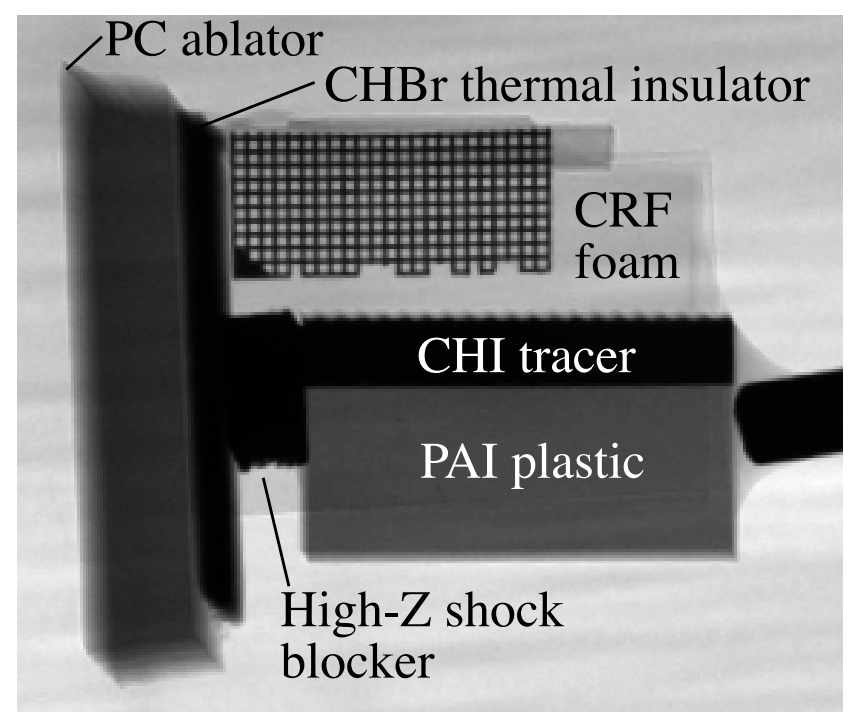

FIG. 1: Radiograph by J. Cowan (LANL) showing the transmission of $\mathrm{Cu}$ x-rays through the different layers of the revised target. The thickness of the PC ablator has been increased from $185 \mu \mathrm{m}$ to $500 \mu \mathrm{m}$. 
The system reaches an Atwood number of $\sim 0.8$ and a convective Mach number of $\sim 0.85$ [14]. The convective Mach number, $M_{c}$, is defined as $\Delta u /\left(c_{1}+c_{2}\right)$, where $\Delta u$ is the relative velocity, and $c$ is the speed of sound. From this definition, in the frame of reference of either fluid, the flow is said to be supersonic at $M_{c} \gtrsim 0.5$. The pressure of the shocked foam was experimentally inferred to be $0.63 \pm 0.08$ Mbar [16].

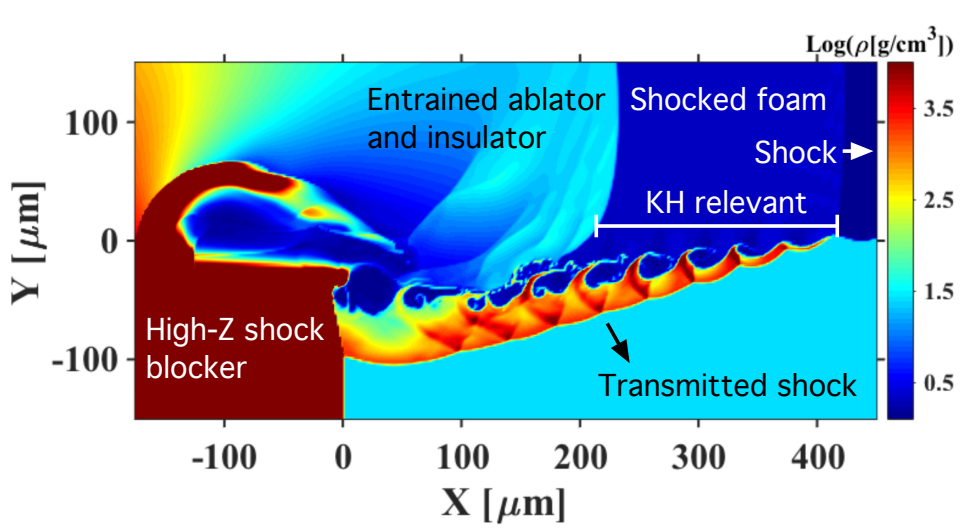

FIG. 2: 2D DAFNA simulation showing a density map of the experimental system at $\mathrm{t}=40 \mathrm{~ns}$. A $20 \mathrm{~ns}$ laser pulse is incident on a $200 \mu \mathrm{m}$ thick PC ablator and 100 $\mu \mathrm{m}$ thick $\mathrm{CHBr}$ thermal insulator. A shockwave is driven through the foam, producing a strong, supersonic shear over a sinusoidal foam-plastic interface.

Figure 2 is a $2 \mathrm{D}$ design simulation of the anticipated system $40 \mathrm{~ns}$ after the laser drive begins [14]. This simulation was produced in DAFNA, a multi-material, HLLCbased Eulerian hydrodynamics code with interface tracking and adaptive mesh refinement capabilities. The simulation is initialized with a 1D Lagrangian radiation hydrodynamics code called HYADES [21]. The materials were treated as ideal gases with an adjustable adiabatic index; in this simulation, the adiabatic index was 1.67 for the CRF foam, and 2.1 for the $\mathrm{PC}$ ablator, $\mathrm{CHBr}$ insulator, and PAI plastic.

In Fig. 2, the shock is moving from the left to the right. As the shock propagates through the foam, a transmitted shock deflects the foam-plastic interface downwards. Along this deflected interface, the modulations begin evolving after the initial shock passes, and the steady, supersonic conditions cease once the thermal insulator that is entrained in the flow disturbs them. As such, the Kelvin-Helmholtz relevant region is said to be what is downstream of the shockwave, yet upstream of the entrained material from the insulator and ablator. Modulations that are immediately behind the shock are in the early stages of evolution, whereas those at the downstream edge have been evolving for several times the characteristic evolutionary timescale of the instability $[14,22]$.

One final laser beam is used to irradiate a $1 \mathrm{~mm}$ diameter, $20 \mu \mathrm{m}$ thick copper foil with $850 \mathrm{~J}$ of $1053 \mathrm{~nm}$ wavelength laser light, focused to a $200 \mu \mathrm{m}$ diameter spot size in a $10 \mathrm{ps}$ pulse, to generate $\mathrm{x}$-rays for a spherical crystal imager diagnostic. The spherical crystal imager was used as an x-ray radiography diagnostic; $\mathrm{x}$-rays prop1.5 agate through the target to a bent crystal, designed to select out the transmission of $\mathrm{Cu} \mathrm{K}_{\alpha}$ radiation $(8.0 \mathrm{keV}$ ) 5 at a $\sim 10$ to $15 \mu \mathrm{m}$ spatial resolution [23], and are then imaged onto a Fuji ${ }^{\mathrm{TM}}$ MS image plate.

\section{RESULTS AND DISCUSSION}

The platform successfully produced the first data of hydrodynamic instability evolution in a steady, supersonic flow from well-characterized initial conditions [16]. However, changes needed to be made to the initial target design to improve the quality and consistency of this data. Figure 3 provides an example of data taken from two different Kelvin-Helmholtz instability campaigns. The first campaign, shown on the left, had a $185 \mu \mathrm{m}$ thick PC ablator and 19 ns laser drive, compared to the $500 \mu \mathrm{m}$ thick $\mathrm{PC}$ ablator and $28 \mathrm{~ns}$ drive in the revised experiment on the right. The images on top are raw radiographs, and the cutouts are samples of the data after they have been processed through a contrast-enhancing unsharp mask algorithm $[14,16,24]$. This algorithm creates a copy of the image, smeared at the diagnostic spatial resolution, and then subtracts this blurred image from the original to locate edges. This information is then combined with the original image to enhance the visibility of those edge features.

One can see from Fig. 3a that while the amplitude of the modulations does change in the original campaign, the modulations do not develop the small-scale rollup structures characteristic to the Kelvin-Helmholtz insta- 

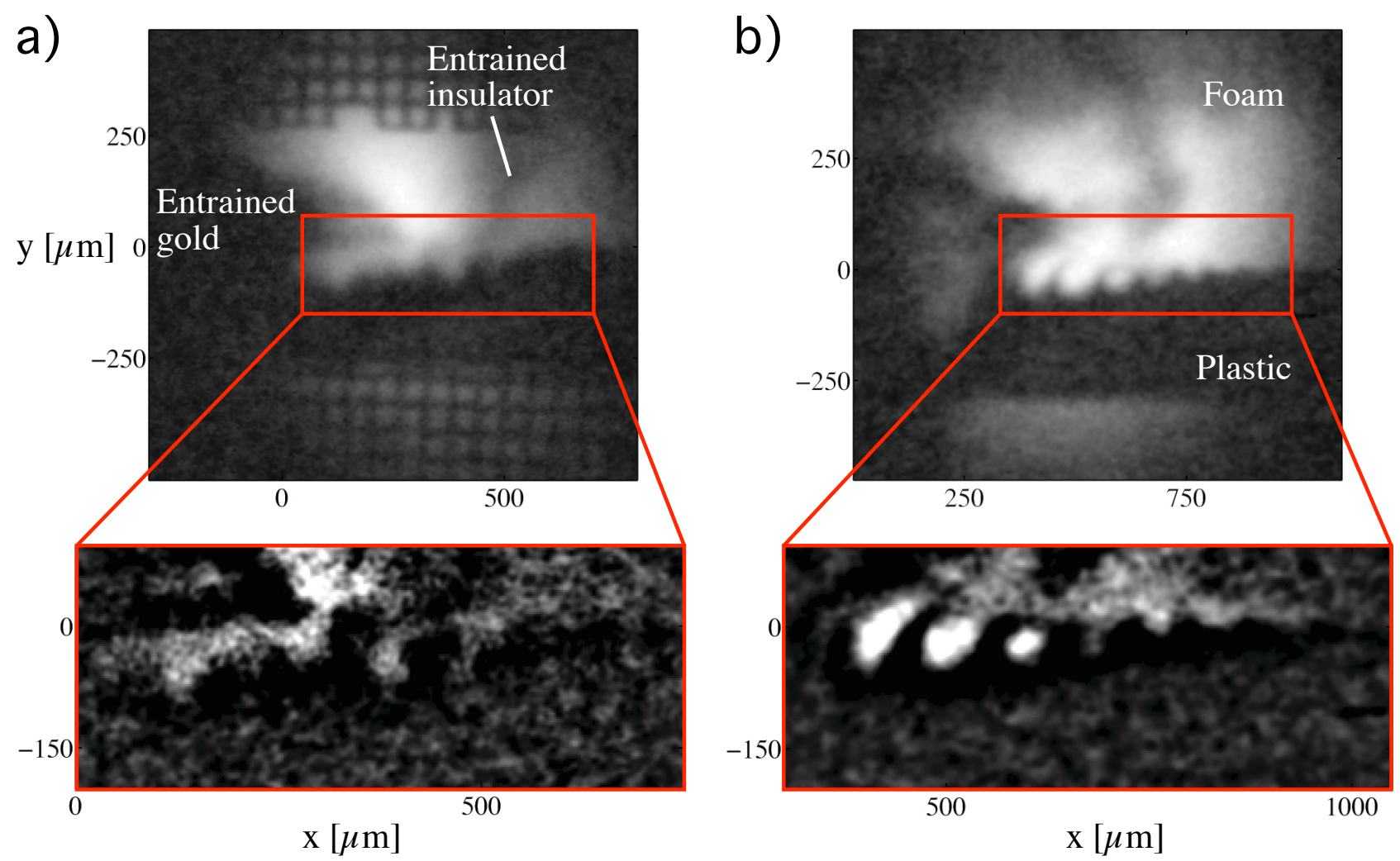

FIG. 3: Left: First experimental campaign with a $185 \mu \mathrm{m}$ thick ablator and 19 ns drive. Right: Second experimental campaign with a $500 \mu \mathrm{m}$ thick ablator and 28 ns drive. Top: Raw radiographic data. Bottom: Contrast-enhanced data.

bility, as seen in the simulation in Fig. 2 [14]. One contributing factor is that the Kelvin-Helmholtz relevant region was smaller than anticipated, resulting in modulations that were disturbed by the entrained insulator and ablator material before they had adequate time to fully develop. This could be due to the foam reaching a higher compression limit than anticipated; in the simulations, the adiabatic index is assumed to be 1.67 for the $\mathrm{CRF}$, resulting in a maximum of a $4 \mathrm{x}$ compression. Firstorder estimates of the experimentally observed foam compression, taken by dividing the distance the shock has traveled through the foam by the length of the KelvinHelmholtz relevant zone, suggests that the foam reached a compression of $\sim 5 \mathrm{x} \pm 1.5[16]$.

The evolution may also have been disturbed by the presence of an unintended secondary shock, which we refer to as a reshock. When the shockwave passes from the thermal insulator into the lower density foam, a rarefac- tion wave is launched back towards the direction of the drive surface. If this expansion wave reaches the critical surface before the laser drive shuts off, then the large pressure gradient is enough to launch a secondary shockwave through the system that can overtake the initial shock and disturb the evolution of the modulations [25]. This reshock can be mitigated or prevented by thickening the ablator package, so as to delay the returning rarefaction wave until the drive pressure has released. Figure 4 demonstrates this with a pair of $1 \mathrm{D}$ radiation hydrodynamic simulations performed with the Hyades code. This code uses the same laser parameters as Fig. 2, but adjusts the initial thicknesses of the $\mathrm{PC}$ ablator. Additionally, the $\mathrm{PC}$ ablator and $\mathrm{CHBr}$ insulator now use polystyrene SESAME equation of state tables instead of an ideal gas model, and the foam uses a carbon foam equation of state table. Various other combinations of equation of state tables or ideal gas adiabatic indices, 
a)

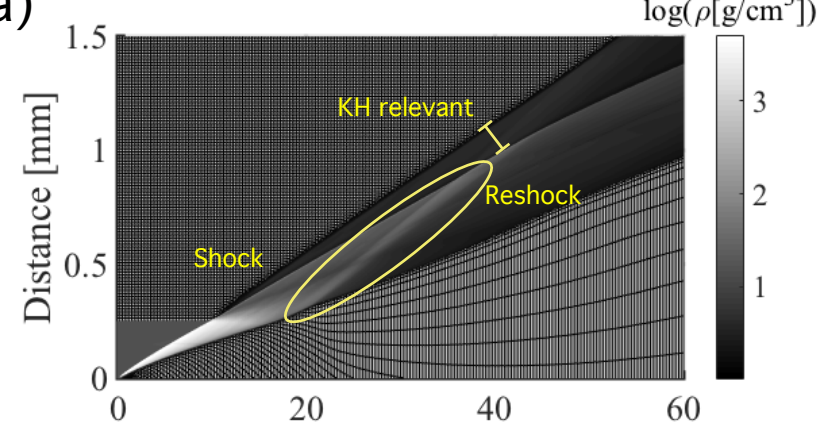

c)

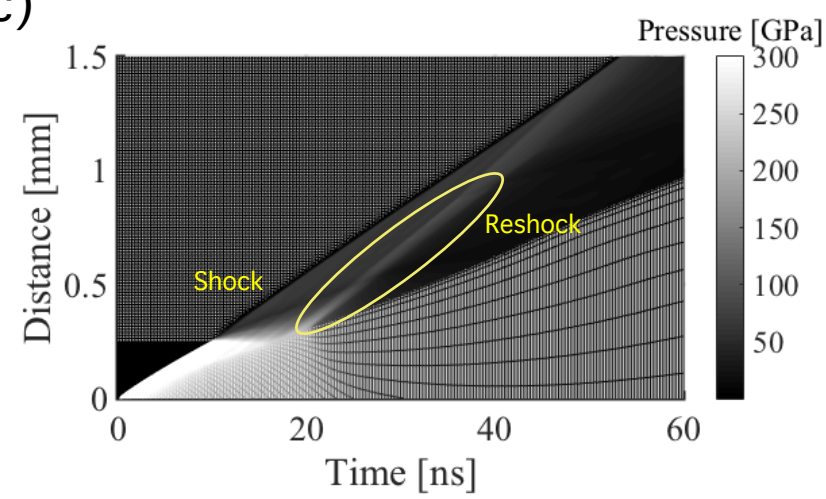

b)

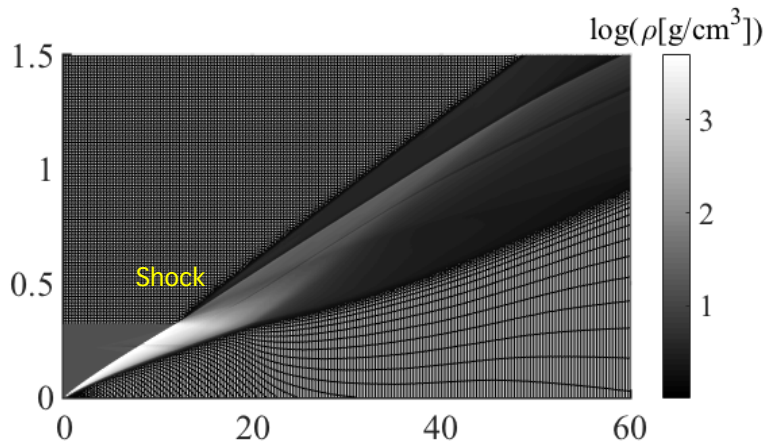

d)

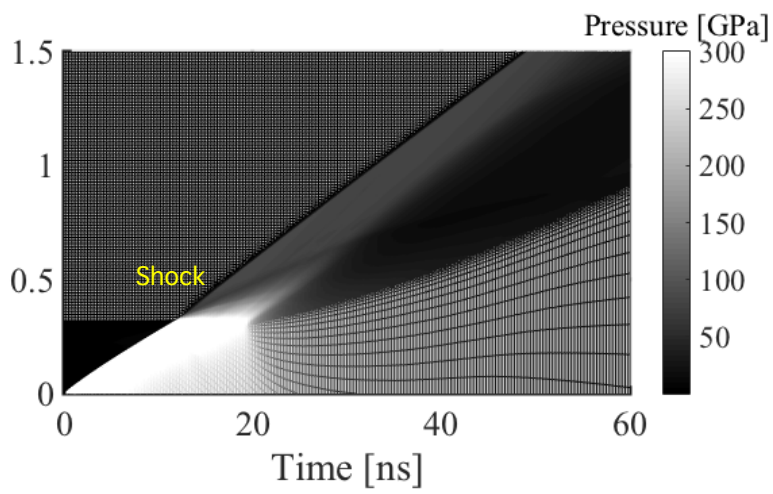

FIG. 4: Density $(\mathrm{a}, \mathrm{c})$ and pressure (b,d) zone - time plots from 1D Hyades simulations. Left: $150 \mu m$ thick PC ablator. Right: $220 \mu m$ thick PC ablator.

ranging from 1.4 to 1.75 , were also explored.

Figure 4 shows density (Fig. 4a, 4c) and pressure (Fig. 4b, 4d) changing with time, with time along the abscissa and distance along the ordinate. In Fig. $4 \mathrm{a}$ and $4 \mathrm{c}$, the initial thickness of the PC ablator is $150 \mu \mathrm{m}$ (35 $\mu \mathrm{m}$ below the nominal experimental thickness), whereas in Fig. $4 \mathrm{~b}$ and $4 \mathrm{~d}$, the initial thickness of the PC ablator is 220 $\mu \mathrm{m}(35 \mu \mathrm{m}$ greater than the nominal experimental thickness). Both are followed by $100 \mu \mathrm{m}$ of $\mathrm{CHBr}$ thermal insulator and $1500 \mu \mathrm{m}$ of CRF foam, and have identical laser conditions. In Fig. 4a and 4c, we can observe the reshock overtaking the initial, driving shockwave, which has the potential to disturb the evolution and fine structure of the shear instability. In Fig. $4 \mathrm{~b}$ and $4 \mathrm{~d}$, the ablator is thick enough to mitigate this secondary shockwave.

The $\pm 35 \mu \mathrm{m}$ in Fig. 4 were chosen for two reasons. First and foremost, a reshock always occurred in these simulations when the ablator thickness was reduced to $35 \mu \mathrm{m}$ below the nominal thickness for all tested combi- nations of equation of state tables and adiabatic indices, while $35 \mu \mathrm{m}$ above the nominal was always observed to be free of a reshock. As a secondary reason, the metrology station we used to perform our pre-experimental evaluation of our target had a $\sim 35 \mu \mathrm{m}$ uncertainty.

Figure 5 presents an alternate view of the same data, with cell number instead of distance along the ordinate. Each cell represents an equivalent mass of the given material, and the transition between materials occurs at predefined cell numbers. Again, the reshock can be seen overtaking the original shockwave in Fig. $5 \mathrm{a}$ and $5 \mathrm{c}$, but is not present in Fig. 5b and 5d.

Depending on which equation of state table or what value adiabatic index is chosen for the various materials, the thickness of the original ablator package may or may not have reached the threshold thickness required prevent this reshock. When we further factor in the variability of individual targets, it is left ambiguous whether or not this reshock may have occurred within the original experiment, and if so, how much of an influence it would 

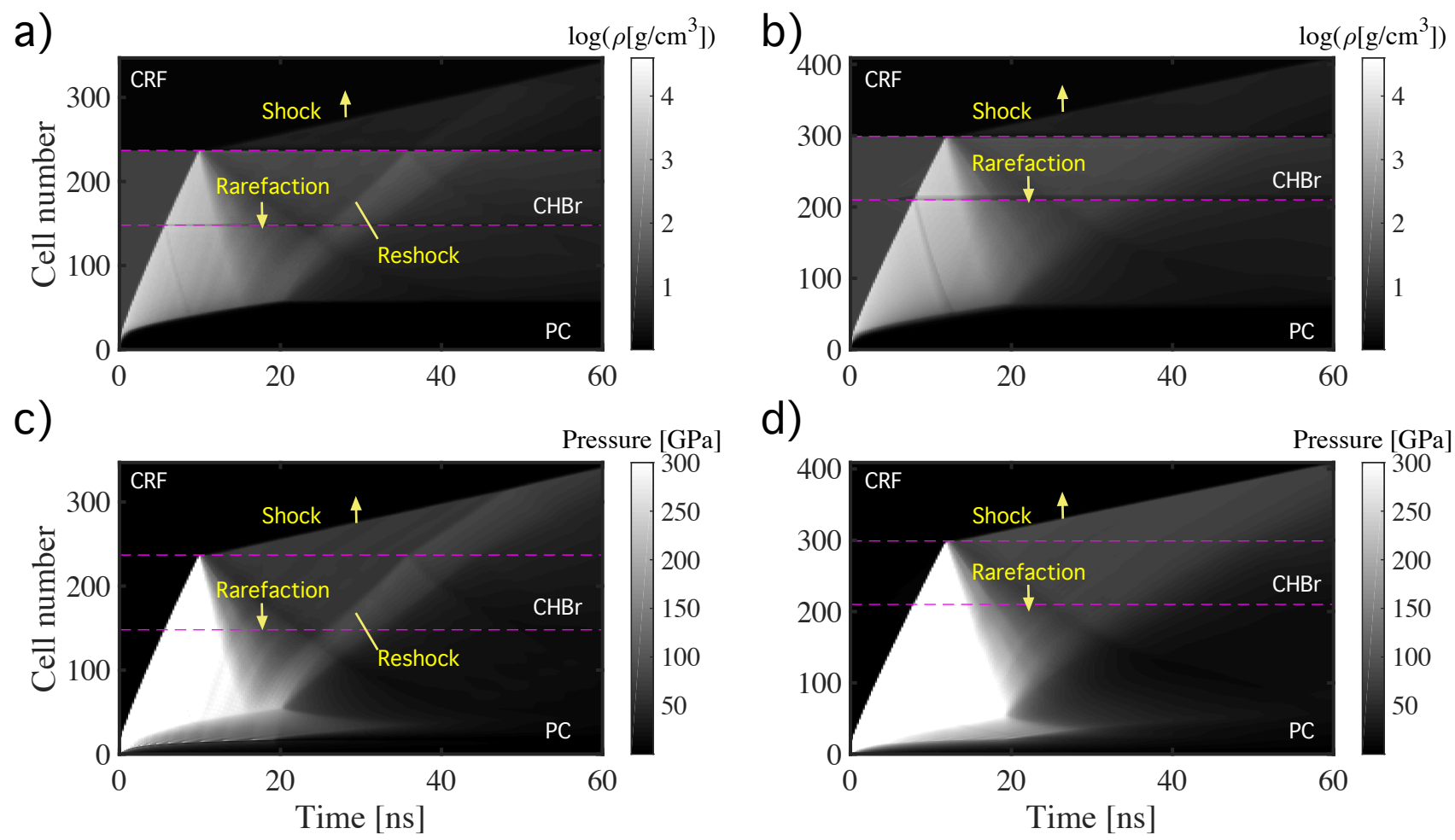

d)

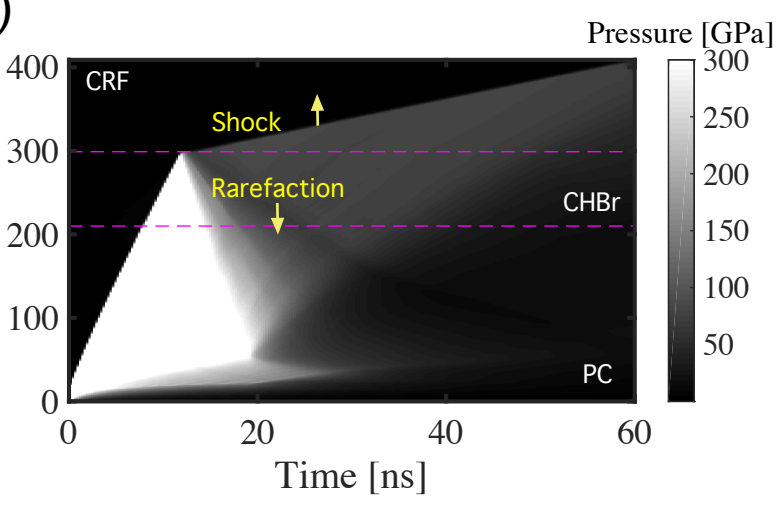

FIG. 5: Density (a,c) and pressure (b,d) cell-number - time plots from 1D Hyades simulations. Each cell contains an equal mass of the specified material. Left: $150 \mu \mathrm{m}$ thick PC ablator. Right: $220 \mu \mathrm{m}$ thick PC ablator.

have had on the development of the instability.

Both concerns, the insufficient size of the KelvinHelmholtz relevant zone and the possibility of a reshock, were addressed in a subsequent campaign by thickening the ablator package and extending the drive duration from 19 ns to $28 \mathrm{~ns}$. Extending the duration of the laser drive extends the maximum duration of the experiment by increasing the length of time over which the shockwave is supported by ablation pressure. This, in turn, provides more time for the primary shockwave to outpace the material that has become entrained in the flow, increasing our instability-relevant region. The ablator thickness was increased to $500 \mu \mathrm{m}$ to compensate for the longer drive duration; this was the thinnest piece that could be ordered from our supplier that still exceeded the threshold thickness needed to eliminate the reshock for all tested combinations of equation of state tables and adiabatic indices. After this change, data and simulation consistently achieved good agreement for large and medium-scale structures [16].
Due to limitations in time and experimental resources, we were unable to perform an experiment in which we changed only the ablator thickness or the laser drive duration exclusively. This remains a topic of interest that can be explored in future experiments. This experiment could also explore the extent to which different types of hydrodynamic instabilities are affected by the reshock (e.g. whether the Rayleigh-Taylor and Kelvin-Helmholtz instabilities are affected to the same degree by the presence of a reshock).

\section{CONCLUSION}

An experimental platform was developed for OMEGAEP to study the evolution of hydrodynamic instabilities in a steady, supersonic flow. An extended laser pulse was used to drive a shockwave over a well-characterized material interface. After analyzing the data from an initial Kelvin-Helmholtz instability experiment, it was determined that we needed better image quality and a 
larger Kelvin-Helmholtz relevant zone. We achieved this by increasing the duration of the laser drive and looking later in time. This change required a thicker ablator, which also addressed the possibility of a reshock that didn't come through in the design stage. Obtaining data later in time also lowered the impact of source effects on the background noise. After these changes, the quality and consistency of the experimental data was significantly improved, and the results were well-reproduced with $2 \mathrm{D}$ simulations [16]. These results demonstrate the experimental platform's ability to produce the first data on the effects of compressibility on the Kelvin-Helmholtz instability's growth rate from single-mode initial conditions. These data, in conjunction with future work, will contribute to the development of a comprehensive model for compressible Kelvin-Helmholtz instability behavior. Additional experiments will use this platform to explore multi-vortex Kelvin-Helmholtz behavior, and extend the platform to study additional hydrodynamic instability behavior.

This work was supported by the U.S. DOE, through NNSA grants DE-NA0002956 (SSAA) and DENA0002719 (NLUF), by the LLE under DE-NA0001944, and by the LLNL under subcontract B614207 to DEAC52-07NA27344.

*Corresponding authors:

W.C. Wan - wwan@umich.edu

G. Malamud - guy.malamud@gmail.com

[1] L. Ofman and B. J. Thompson. Sdo/aia observation of kelvin-helmholtz instability in the solar corona. The Astrophysical Journal Letters, 734(1):L11, 2011.

[2] P. A. Delamere, R. J. Wilson, S. Eriksson, and F. Bagenal. Magnetic signatures of kelvin-helmholtz vortices on saturn's magnetopause: Global survey. Journal of Geophysical Research, 118:303-404, Jan 2013.

[3] Carl L. Gardner and Steven J. Dwyer. Numerical simulation of the $\{\mathrm{XZ}\}$ tauri supersonic astrophysical jet. Acta Mathematica Scientia, 29(6):1677 - 1683, 2009.

[4] A. I. MacFadyen. Long GRBs and Supernovae from Collapsars. In J.-M. Marcaide and K. W. Weiler, editors, IAU Colloq. 192: Cosmic Explosions, On the 10th Anniversary of SN1993J, page 417, 2005.

[5] M. Itoh, S. Kumagai, T. Shigeyama, K. Nomoto, and J. Nishimura. X-rays expected from supernova 1987A compared with the source discovered by the GINGA satellite. Nature (London), 330:233-235, November 1987.
[6] T. Ebisuzaki, T. Shigeyama, and K. Nomoto. RayleighTaylor instability and mixing in SN 1987A. Astrophysical Journal, 344:L65-L68, September 1989.

[7] A. B. Zylstra, J. A. Frenje, F. H. Sguin, D. G. Hicks, E. L. Dewald, H. F. Robey, J. R. Rygg, N. B. Meezan, M. J. Rosenberg, H. G. Rinderknecht, S. Friedrich, R. Bionta, R. Olson, J. Atherton, M. Barrios, P. Bell, R. Benedetti, L. Berzak Hopkins, R. Betti, D. Bradley, D. Callahan, D. Casey, G. Collins, S. Dixit, T. Döppner, D. Edgell, M. J. Edwards, M. Gatu Johnson, S. Glenn, S. Glenzer, G. Grim, S. Hatchett, O. Jones, S. Khan, J. Kilkenny, J. Kline, J. Knauer, A. Kritcher, G. Kyrala, O. Landen, S. LePape, C. K. Li, J. Lindl, T. Ma, A. Mackinnon, A. Macphee, M. J.-E. Manuel, D. Meyerhofer, J. Moody, E. Moses, S. R. Nagel, A. Nikroo, A. Pak, T. Parham, R. D. Petrasso, R. Prasad, J. Ralph, M. Rosen, J. S. Ross, T. C. Sangster, S. Sepke, N. Sinenian, H. W. Sio, B. Spears, P. Springer, R. Tommasini, R. Town, S. Weber, D. Wilson, and R. Zacharias. The effect of shock dynamics on compressibility of ignition-scale national ignition facility implosions. Physics of Plasmas, 21(11):112701, 2014.

[8] J. D. Moody, D. A. Callahan, D. E. Hinkel, P. A. Amendt, K. L. Baker, D. Bradley, P. M. Celliers, E. L. Dewald, L. Divol, T. Döppner, D. C. Eder, M. J. Edwards, O. Jones, S. W. Haan, D. Ho, L. B. Hopkins, N. Izumi, D. Kalantar, R. L. Kauffman, J. D. Kilkenny, O. Landen, B. Lasinski, S. LePape, T. Ma, B. J. MacGowan, S. A. MacLaren, A. J. Mackinnon, D. Meeker, N. Meezan, P. Michel, J. L. Milovich, D. Munro, A. E. Pak, M. Rosen, J. Ralph, H. F. Robey, J. S. Ross, M. B. Schneider, D. Strozzi, E. Storm, C. Thomas, R. P. J. Town, K. L. Widmann, J. Kline, G. Kyrala, A. Nikroo, T. Boehly, A. S. Moore, and S. H. Glenzer. Progress in hohlraum physics for the national ignition facility). Physics of Plasmas (1994-present), 21(5):056317, 2014.

[9] E. E. Meshkov. Instability of the interface of two gases accelerated by a shock wave. Fluid Dynamics, 4(5):101$104,1969$.

[10] Jacob McFarland, David Reilly, Skylar Creel, Christopher McDonald, Thomas Finn, and Devesh Ranjan. Experimental investigation of the inclined interface richtmyer-meshkov instability before and after reshock. Experiments in Fluids, 55(1):1-14, 2013.

[11] P. R. Chapman and J. W. Jacobs. Experiments on the three-dimensional incompressible richtmyer-meshkov instability. Physics of Fluids, 18(7), 2006.

[12] E. C. Harding, J. F. Hansen, O. A. Hurricane, R. P. Drake, H. F. Robey, C. C. Kuranz, B. A. Remington, M. J. Bono, M. J. Grosskopf, and R. S. Gillespie. Observation of a kelvin-helmholtz instability in a high-energydensity plasma on the omega laser. Phys. Rev. Lett., 103:045005, Jul 2009.

[13] O.A. Hurricane. Design for a high energy density kelvinhelmholtz experiment. High Energy Density Physics, 4(34):97 - 102, 2008.

[14] G. Malamud, A. Shimony, W.C. Wan, C.A. Di Stefano, Y. Elbaz, C.C. Kuranz, P.A. Keiter, R.P. Drake, and D. Shvarts. A design of a two-dimensional, supersonic kh experiment on omega-ep. High Energy Density Physics, 9(4):672 - 686, 2013.

[15] C. A. Di Stefano, G. Malamud, C. C. Kuranz, S. R. Klein, C. Stoeckl, and R. P. Drake. Richtmyer-meshkov evolution under steady shock conditions in the high-energy- 
density regime. Applied Physics Letters, 106(11), 2015.

[16] W. C. Wan, G. Malamud, A. Shimony, C. A. Di Stefano, M. R. Trantham, S. R. Klein, D. Shvarts, C. C. Kuranz, and R. P. Drake. Observation of single-mode, kelvinhelmholtz instability in a supersonic flow. Phys. Rev. Lett., 115:145001, Oct 2015.

[17] W. Thomson (Lord Kelvin). Hydrokinetic solutions and observations. The London, Edinburgh, and Dublin Philosophical Magazine and Journal of Science, Philosophical Magazine Series 4, 42(281):362-377, 1871.

[18] H. Helmholtz. Über discontinuierliche flüssigkeitsbewegungen (on the discontinuous movements of fluids). Monatsberichte der Koniglichen Preussiche Akademie der Wissenschaften zu Berlin [Monthly Reports of the Royal Prussian Academy of Philosophy in Berlin], Philosophical Magazine Series 4, 36(244):337-346, 1868.

[19] S.Roy Choudhury. Nonlinear evolution of the kelvinhelmholtz instability of supersonic tangential velocity discontinuities. Journal of Mathematical Analysis and Applications, 214(2):561 - 586, 1997.

[20] D N Maywar, J H Kelly, L J Waxer, S F B Morse, I A Begishev, J Bromage, C Dorrer, J L Edwards, L Folnsbee, M J Guardalben, S D Jacobs, R Jungquist, T J Kessler, R W Kidder, B E Kruschwitz, S J Loucks, J R Marciante, R L McCrory, D D Meyerhofer, A V Okishev,
J B Oliver, G Pien, J Qiao, J Puth, A L Rigatti, A W Schmid, M J Shoup III, C Stoeckl, K A Thorp, and J D Zuegel. Omega ep high-energy petawatt laser: progress and prospects. Journal of Physics: Conference Series, 112(3):032007, 2008.

[21] Jon T. Larsen and Stephen M. Lane. Hyades - plasma hydrodynamics code for dense plasma studies. Journal of Quantitative Spectroscopy and Radiative Transfer, 51(12):179 - 186, 1994. Special Issue Radiative Properties of Hot Dense Matter.

[22] U. Frisch. Phenomenology of turbulence in the sense of kolmogorov 1941. In Turbulence: the legacy of A.N. Kolmogorov. Cambridge University Press., Cambridge, 1995.

[23] C. Stoeckl, G. Fiksel, D. Guy, C. Mileham, P. M. Nilson, T. C. Sangster, M. J. Shoup, and W. Theobald. A spherical crystal imager for omega ep. Review of Scientific Instruments, 83(3):033107, 2012.

[24] C.A. Di Stefano, G. Malamud, C.C. Kuranz, S.R. Klein, and R.P. Drake. Measurement of richtmyer-meshkov mode coupling under steady shock conditions and at high energy density. High Energy Density Physics, 17, Part B:263 - 269, 2015.

[25] R.P. Drake. Reshocks in rarefactions. In Y. Horie L. Davison, editor, High-Energy-Density Physics. Springer, New York, 2006. 